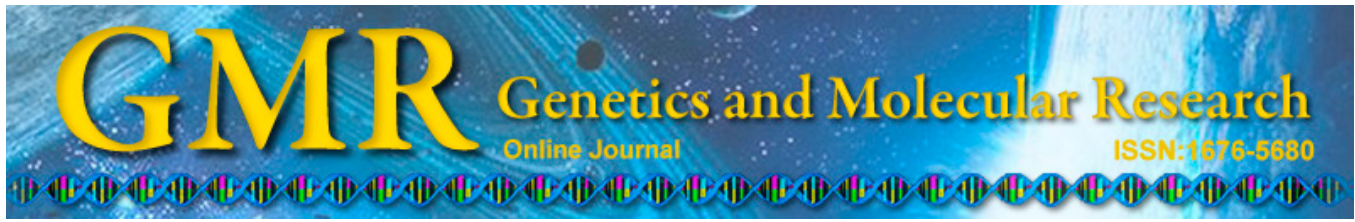

\title{
Isolation of the P5CS gene from reed canary grass and its expression under salt stress
}

\author{
L.L. Cong ${ }^{1,2,3}$, X.Q. Zhang ${ }^{3}$, F.Y. Yang ${ }^{1}$, S.J. Liu' ${ }^{1}$ and Y.W. Zhang ${ }^{1}$ \\ ${ }^{1}$ Institute of Grassland Science, China Agricultural University, \\ Beijing, China \\ ${ }^{2}$ Institute of Animal Sciences, Chinese Academy of Agricultural Sciences, \\ Beijing, China \\ ${ }^{3}$ Department of Grassland Science, Sichuan Agricultural University, \\ Ya'an, China \\ Corresponding author: Y.W. Zhang \\ E-mail: zywei@cau.edu.cn
}

Genet. Mol. Res. 13 (4): 9122-9133 (2014)

Received September 30, 2013

Accepted August 1, 2014

Published October 31, 2014

DOI http://dx.doi.org/10.4238/2014.October.31.28

\begin{abstract}
Reed canary grass (RCG) is a perennial grass traditionally cultivated for forage. It is also used as fuel to produce energy in Finland and Sweden, and other countries have expressed interest in the cultivation of RCG. In China, arable land is limited. Salinity is considered to be a major factor limiting plant crop development and productivity. To boost biofuel production of RCG and extend its range in saline soil, we seek to improve its salt tolerance. Proline acts as an osmolyte that accumulates when plants are subjected to abiotic stress. $P 5 C S$ plays a crucial role in proline biosynthesis. We isolated a P5CS gene from RCG, designated B231P5CS (GenBank accession No. JQ622685). B231P5CS is a fragment (971 bp) that encodes a 323-amino acid polypeptide. We also cloned an actin gene fragment from RCG as a reference gene in expression analysis of B231P5CS gene. Expression analysis revealed that $B 231 P 5 C S$ transcripts were upregulated in leaves after treatment with salt $(200 \mathrm{mM} \mathrm{NaCl})$ and that transcript levels of $B 231 P 5 C S$ reached a maximum $12 \mathrm{~h}$ after exposure, which was 14.69
\end{abstract}


times the level in control plants. The trends of expression were exactly opposite in roots; transcripts were downregulated after salt treatment. Proline concentration increased in leaves after stress. In contrast, proline content of roots decreased up to 3.6-fold relative to controls. Changes in proline concentration after stress were correlated with B231P5CS expression. Our results suggest that B231P5CS is a stress-inducible gene and plays a non-redundant role in plant development. This gene may be used to improve stress tolerance of RGC and other bioenergy feedstock.

Key words: Reed canary grass; P5CS; Gene clone; Salt stress

\section{INTRODUCTION}

Reed canary grass (RCG, Phalaris arundinacea L.), a native grass in northern temperate regions, is widely distributed throughout Europe, Asia, America, and Africa (Sahramaa, 2004). RCG is a perennial grass that is traditionally cultivated for forage but is also a potential crop for bio-energy and paper pulp production in northern regions (Lewandowski et al., 2003; Finell and Nilsson, 2005). Furthermore, replacing annual food crops on arable land with perennial energy crops is beneficial to the environment, as it increases soil fertility and reduces soil erosion, nutrient leaching, heavy metal content in the soil, and the emission of greenhouse gases from soil leaching (Borjesson, 1999). Because of its high biomass yield and persistence under wet and dry conditions, RCG has become an attractive field crop. Relative to other gramineous forage crops, RCG has low maintenance requirements, strong resistance, high wood fiber content, and easy management. It is a renewable energy source that can be harvested for 10-12 years after the establishment of its cultivation in northern climates (Vepsalainen, 2010). RCG is considered to be the most suitable energy crop for northern Scandinavian weather conditions (Landstrom et al., 1996). In recent years, RCG has been used on a large scale for energy purposes in Finland and Sweden. Other European countries have also shown interest in the cultivation of RCG (Landstrom et al., 1996; Sahramaa and Jauhiainem, 2003; Anderson and Fergusson, 2006). In Finland, approximately $1 \%$ of the agricultural area is under RCG cultivation. Moreover, the Finnish Ministry of Forestry and Agriculture has set the official goal to increase RCG cultivation fivefold by the year 2015 (Vepsalainen, 2010), which would mean that the RCG area would exceed the recent combined area of potato, turnip rape, rape, and sugar beet.

Salinization of soil is a serious problem and its area is increasing steadily in many parts of the world, particular in arid and semi-arid areas (Latef, 2010). At present, out of 1.5 billion hectares of cultivated land around the world, about 77 million hectares (5\%) are affected by excess salt content (Sheng et al., 2008). Development of salt-resistant cultivars is a high priority for many crop breeding programs worldwide. Agronomic and crop management strategies aim at expanding the range of crop growth to marginal or barren soils, especially saline-alkaline soils. These strategies include crop rotation and tillage practices. However, these have generally not been highly effective.

Previously, we studied seed germination and the physiological and biochemical characteristics of RCG seedlings subjected to simulated salt stress to evaluate salt tolerance and the physiological changes that occur under various levels of salt stress. Using a salt-tolerant identification test, we found that Zxy06p-2449 was the most salt tolerant and all germplasms 
were most sensitive to salt concentrations of $200 \mathrm{mM}$. We also found that of the measured physiological indicators, proline content had the greatest change in response to salt stress. Proline accumulation is a common metabolic response in higher plants that are exposed to high-salt conditions. Proline acts as an osmolyte that not only stabilizes protein structures, but also acts as a regulator of cellular redox potential (Verbruggen and Hermans, 2008; Szabados and Savoure, 2009). The ability to accumulate proline under stress is often associated with stress tolerance in various plant species (Anoop and Gupta, 2003; Hmida-Sayari et al., 2005). Proline is synthesized by either the glutamate or arginine/ornithine pathways in higher plants; the glutamate pathway is the major route for proline synthesis during the stress-response process (Zhang et al., 1995). Under the glutamate pathway, proline is synthesized from glutamic acid via the intermediates $\gamma$-glutamic semialdehyde and $\Delta 1$-pyrroline-5-carboxylate (P5C). P5C synthetase (P5CS), which catalyzes the first two reactions of proline biosynthesis, is a bifunctional enzyme with both $\gamma$-glutamyl kinase and the glutamic- $\gamma$-semialdehyde dehydrogenase activities. The $\gamma$-glutamyl kinase activity of P5CS represents the rate-limiting step in this pathway and also is subject to feedback inhibition by proline (Zhang et al., 1995). P5CS genes have been isolated from several plant species. A P5CS gene was first cloned from Vigna aconitifolia (Hu et al., 1992). The upregulation of P5CS expression and the accumulation of proline have a cause-and-effect relationship in Oryza sativa (Hur et al., 2004; Sripinyowanich et al., 2013) and Arabidopsis thaliana (Savoure et al., 1995).

RCG has been recognized as a promising energy plant, due to its high yield. RCG can also be used as livestock fodder. However, the development of bioenergy feedstock is limited by the high cost of arable land. If bioenergy plants could be grown on saline-alkaline soils, the twofold problem of feedstock production and rural poverty could be addressed. Salt tolerance seems to be a key target for expanding this crop to marginal soils. Conventional breeding is time-consuming and laborious. Genetic engineering is an alternative approach to the generation of salt-tolerant plants. The functions of P5CS make it a candidate for genetic engineering. To target a gene for overexpression, we need to know the endogenous expression patterns of P5CS genes. In this study, the cDNA of P5CS was cloned from RCG. Expression levels of the $P 5 C S$ gene were analyzed in the leaves and roots of RCG seedlings that were exposed to salt stress $(200 \mathrm{mM})$.The relationship between the accumulation of proline and the upregulation of P5CS expression was also demonstrated. The results of this study suggest that the P5CS gene could be used as a target for the genetic engineering of RCG to improve salt stress tolerance.

\section{MATERIAL AND METHODS}

\section{Plant materials and stress treatments}

Seeds of RCG (Zxy06p-2449) originating from Russia were obtained from the Gao Hongwen group (Institute of Animal Sciences, Chinese Academy of Agricultural Sciences). In this experiment, full seeds were selected and sterilized in $3 \% \mathrm{H}_{2} \mathrm{O}_{2}$ for $10 \mathrm{~min}$, then rinsed in running water followed by distilled water. Seeds were then planted in plastic pots, which were filled with $1.7 \mathrm{~L}$ distilled water (Figure 1A). After germination, seedlings were transferred to pots containing half-strength Hoagland's solution (Figure 1B and C), and raised in an intelligent greenhouse under controlled conditions $\left(10 \mathrm{~h}\right.$ light $/ 14 \mathrm{~h}$ dark at $\left.25^{\circ} \pm 2{ }^{\circ} \mathrm{C}\right)$. After 30 days (Figure 1D), a total of 21 pots with 210 plants were subjected to a salt treatment (200 mM $\mathrm{NaCl}$ prepared in half-strength Hoagland's solution). Leaf and root samples were collected at 
$0,2,4,8,12,24$, and $48 \mathrm{~h}$ after the initiation of treatment. Samples were snap-frozen in liquid nitrogen. The frozen material was then used for total mRNA extraction and determination of proline content.

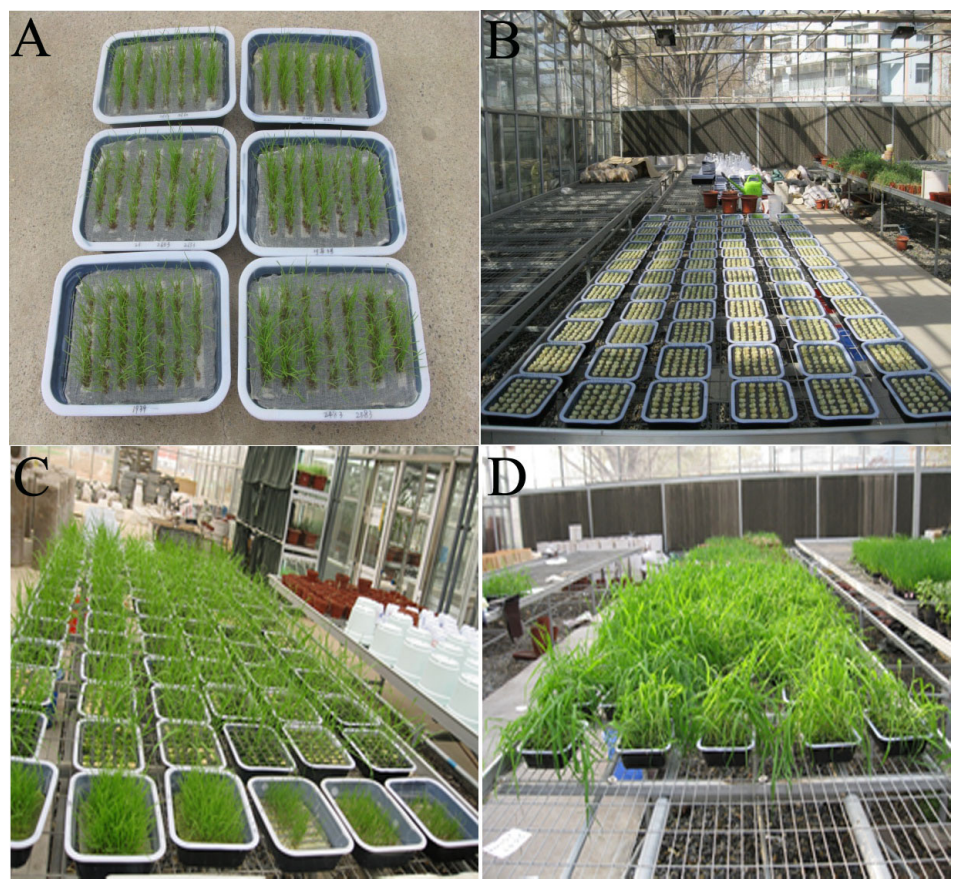

Figure 1. A. Germination of reed canary grass (Phalaris arundinacea L.). B. Seedlings were transferred to pots. C. Seedlings after 10 days of growth. D. After 30 days of growth, seedlings were treated with $200 \mathrm{mM} \mathrm{NaCl}$.

\section{Isolation of total RNA and synthesizing first strand cDNA}

Total RNA was extracted using an RNAprep Pure Plant kit (TIANGEN BIOTECH Co., Ltd, China). A 1- $\mu$ g sample of total RNA was used for first-strand cDNA synthesis using a TIANscript RT Kit RT-PCR kit (TIANGEN BIOTECH Co., Ltd., China), according to manufacturer instructions.

\section{Cloning P5CS cDNA and sequence analysis}

For amplification of the $P 5 C S$ gene from $\mathrm{RCG}$, a pair of primers $\left(\mathrm{P}_{1}-\mathrm{F}\right.$ : 5'-GCCAGAA GATAGCAAG-3'; P - R: 5'-GACCTTCAACACCCACAG-3') was designed based on the conserved region sequence of the P5CS gene from several plants. The $25-\mu \mathrm{L}$ PCR mixture contained $1 \mu \mathrm{L}$ cDNA template, $12.5 \mu \mathrm{L}$ PCR mix, $10.5 \mu \mathrm{LddH}_{2} \mathrm{O}$, and $0.5 \mu \mathrm{M}$ primers. The PCR program was set up as follows: denaturation at $94^{\circ} \mathrm{C}$ for $10 \mathrm{~min}, 35$ cycles of $94^{\circ} \mathrm{C}$ for $30 \mathrm{~s}, 54^{\circ} \mathrm{C}$ for $30 \mathrm{~s}$ and $72^{\circ} \mathrm{C}$ for $60 \mathrm{~min}$, followed by final extension at $72^{\circ} \mathrm{C}$ for $5 \mathrm{~min}$. The fragments obtained were sub-cloned using a pUC-T vector (Huayueyang Corporation, China), and then transferred to DH5 $\alpha$ competent cells (Huayueyang Corporation). The positive clones 
were subjected to M13 primer-based Sanger sequencing. Sequences were aligned using DNAMAN and BLAST on-line. A neighbor-joining method implemented in ClustalW and MEGA 5.0 were used to construct phylogenetic trees of the P5CS protein.

\section{Actin gene clone}

As the success of RT-qPCR depends on the stability of the reference gene(s) used for data normalization, we isolated a beta-actin gene fragment to use as the reference gene. To amplify the actin gene from RCG, we used a homologous actin gene from Lolium perenne (GenBank accession No.: AY014278.1) that was highly homologous to the actin genes obtained from other plant species contained in the NCBI BLAST program. A set of primers (sense: F5'-GACCGAGCGTGGTTACTC-3', antisense: 5'-GCACCTCCAATCCAGACA-3') were designed based on this coding sequence. RT-PCR was conducted under the following conditions: $94^{\circ} \mathrm{C}$ for $4 \mathrm{~min}, 35$ cycles $\left(94^{\circ} \mathrm{C}\right.$ for $30 \mathrm{~s}, 54^{\circ} \mathrm{C}$ for $30 \mathrm{~s}$ and $72^{\circ} \mathrm{C}$ for $1 \mathrm{~min}$ ), followed by $72^{\circ} \mathrm{C}$ for $5 \mathrm{~min}$. The PCR products of the predicted size (approx. $450 \mathrm{bp}$ ) were purified and cloned in a pUC-T vector (Huayueyang Corporation) and then sequenced by Shanghai Invitrogen Biotechnology Co., Ltd. (China).

\section{Real time PCR analysis}

Total RNA isolation and first-strand cDNA synthesis were performed under the same conditions as used for cloning the P5CS gene. The following PCR primers were used for B231P5CS: RP-F: 5'-TAATGTTGGAAGAGGTGGC-3' and RP-R: 5'-CAAGGCCCCACTCTTGT-3'. The actin primers used were RA-F: 5'-ACAGAGACAAAGTAGCAGAG-3' and RA-R: 5'-AGCACCCAGTCCAAAACGAG-3'. RT-qPCR was carried out on 96-well plates using a Bio-Rad Real-Time PCR System and a SYBR Green-based PCR assay. Each reaction mix contained $1 \mu \mathrm{L}$ diluted cDNA, $10 \mu \mathrm{L}$ Maxima ${ }^{\circledR}$ SYBR Green/ROX qPCR Master Mix, $0.4 \mu \mathrm{M}$ of each primer to a final volume of $20 \mu \mathrm{L}$. The reaction mix was subjected to the following conditions: $95^{\circ} \mathrm{C}$ for $10 \mathrm{~min}$, followed by 45 cycles of $95^{\circ} \mathrm{C}$ for $15 \mathrm{~s}$ and $60^{\circ} \mathrm{C}$ for $30 \mathrm{~s}$. The melting curves were then analyzed at $95^{\circ} \mathrm{C}$ for $15 \mathrm{~s}, 60^{\circ} \mathrm{C}$ for $15 \mathrm{~s}$, and $95^{\circ} \mathrm{C}$ for $15 \mathrm{~s}$. In addition, a reverse transcription negative control was included to check for potential genomic DNA contamination. Each RT-qPCR analysis was performed in triplicate and the mean was used for RT-qPCR analysis. The relative expression of the B231P5CS gene was calculated according to the $2^{-\Delta \Delta C t}$ method (Livak and Schmittgen, 2001). The threshold cycle (CT) values for both the target and the internal control genes were the means of triplicate independent PCRs.

\section{Free proline levels}

Free proline was extracted and quantified according to the method described by Magne and Larher (1992). Briefly, $0.5 \mathrm{~g}$ tissue was homogenized in 3\% sulfosalicylic acid, boiled in water for $10 \mathrm{~min}$, chilled on ice, and then centrifuged at 12,000 $\mathrm{g}$ for $10 \mathrm{~min}$. Two milliliters of supernatant were then mixed with $3 \mathrm{~mL} 2.5 \%$ ninhydrin and $3 \mathrm{~mL}$ acetic acid. The mixture was boiled for $1 \mathrm{~h}$, cooled gradually, and then extracted using $5 \mathrm{~mL}$ toluene. Absorbance was measured at $520 \mathrm{~nm}$ using a UV visible spectrophotometer. Appropriate proline standards were included for the calculation of proline concentration in the samples. 


\section{Statistical analysis}

Data are reported as the means $\pm \mathrm{SE}$ for each treatment $(\mathrm{N}=3)$. Analysis of variance was performed using SPSS 15.0 (SPSS Inc, Chicago, IL, USA). The significance of differences between means was calculated using the Duncan multiple range test $(\mathrm{P}<0.05)$.

\section{RESULTS}

\section{B231P5CS sequence analysis and phylogenetic tree construction}

A 971-bp amplification fragment was identified from RT-PCR (Figure 2A) after the selection of white colonies. Bacterial culture broth PCR confirmed the presence of this fragment (Figure 2B). The P5CS gene encoded 323 amino acid polypeptides (Figure 3). It was named $B 231 P 5 C S$ and registered in NCBI GenBank (Accession No. JQ622685). Sequence analysis showed that B231P5CS was $76 \%$ homologous to SbP5CS2 in nucleotide sequence, and had high homology with wheat (93\%) and rice (88\%). The gene sequence and deduced amino acid sequences are shown in Figure 3. To analyze the evolutionary relationships of the B231P5CS gene within an array of plant P5CSs, a phylogenetic tree was deduced from the amino acid sequence. Three major groups were distinguished within the tree (Figure 4). B231P5CS belonged to group II, indicating that it has a relatively close evolutionary relationship with Puccinellia chinampoensis and Triticum aestivum genetic. Overall, these observations indicated that B231P5CS shares common features with P5CSS obtained from other plants.

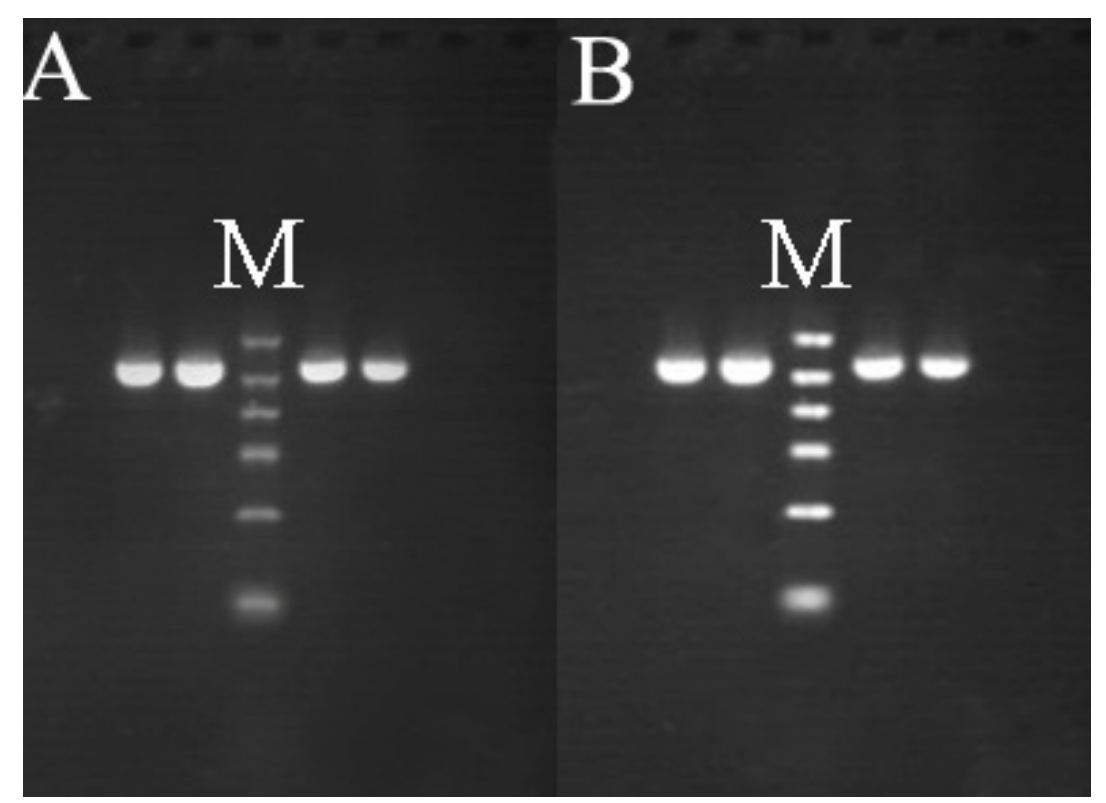

Figure 2. Amplification of the P5CS gene from a cDNA fragment (A) and identification of P5CS by PCR of the bacterial culture broth (B). Lane $M=$ DNA marker II $(100,300,500,700,900$, and $1200 \mathrm{bp})$. 


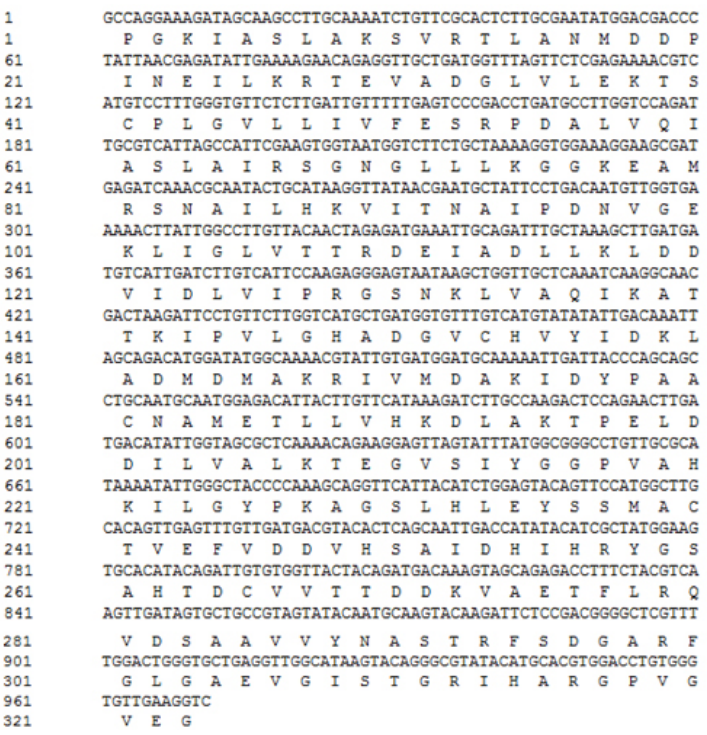

Figure 3. B231P5CS gene sequence and deduced amino acid sequence.

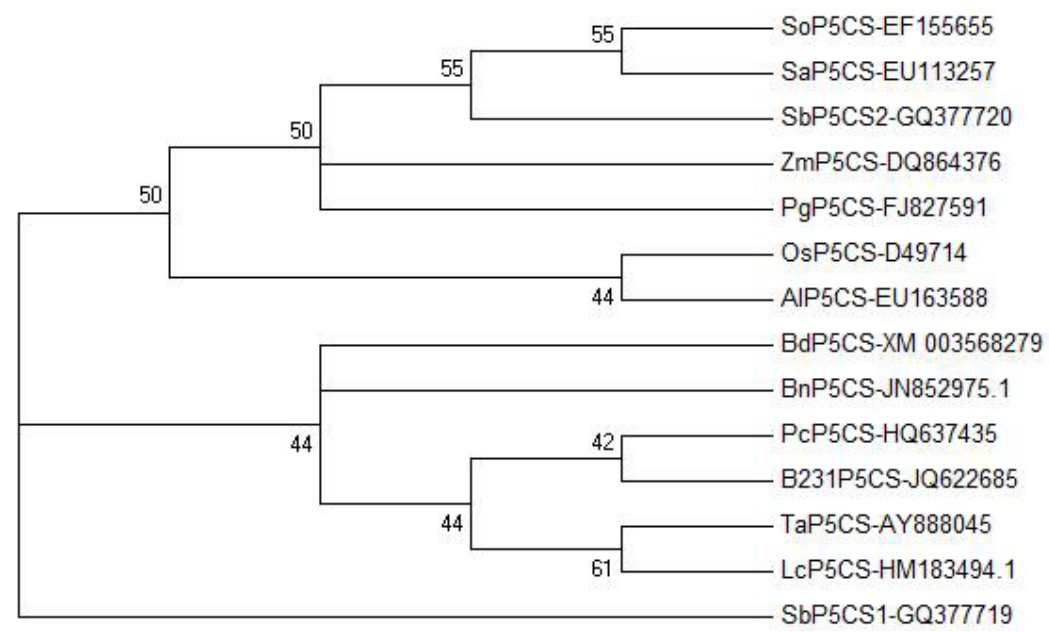

Figure 4. Phylogenetic relationships of B231P5CS with PcP5CS (Puccinellia chinampoensis P5CS), SoP5CS (Saccharum officinarum P5CS), OsP5CS (Oryza sativa P5CS), SbP5CS1 (Sorghum bicolor P5CS1), SbP5CS2 (S. bicolor P5CS2), TaP5CS (Triticum aestivum P5CS), ZmP5CS (Zea mays P5CS), AlP5CS (Aeluropus lagopoides P5CS), BdP5CS (Brachypodium distachyon P5CS), SaP5CS (Saccharum arundinaceum P5CS), PgP5CS (Pennisetum glaucum P5CS), BnP5CS (Boehmeria nivea P5CS), and LcP5CS (Leymus chinensis P5CS). Three groups are represented and B231P5CS is in Group II, as indicated in bold.

\section{Actin gene clone}

A 450-bp cDNA fragment coding for actin protein was amplified using homology cloning methods (Figure 5A) after the selection of white colonies, and then bacterial culture broth PCR further identified the gene (Figure 5B). cDNA of actin that encodes 150-amino acid polypeptides was isolated from RCG sequences are shown in Figure 6. 


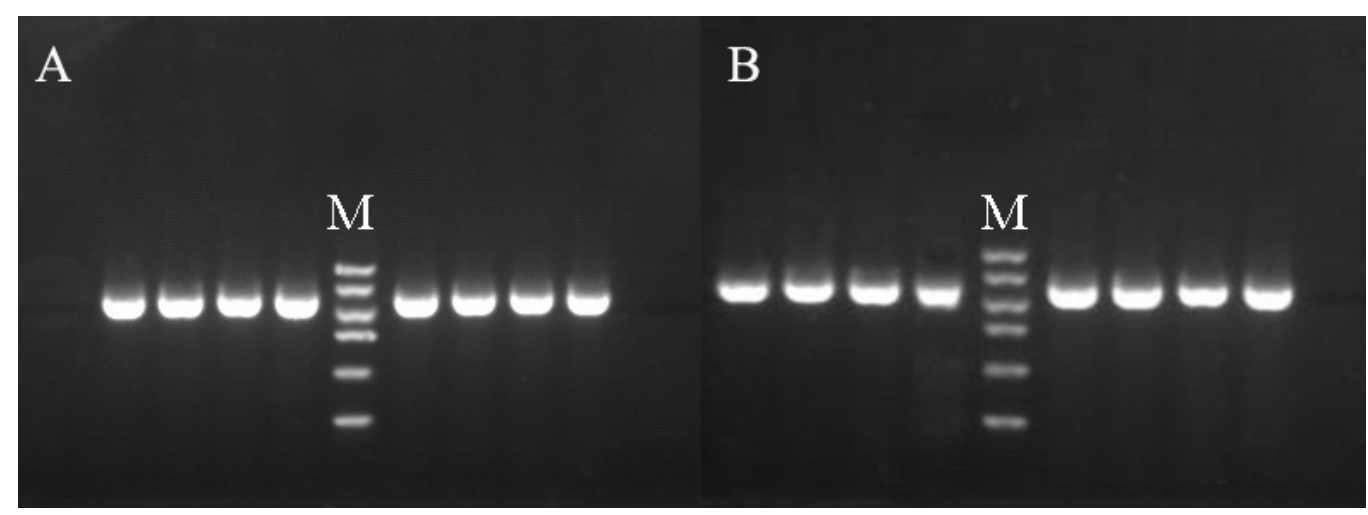

Figure 5. Amplified results of an actin gene fragment (A) and identification of actin using bacterial culture broth PCR (B). Lane $M=$ DNA marker II (100, 300, 500, 700, 900, and 1200 bp).

GACCGAGCGT GGTTACTCAT TCACCACCAC GGCCGAGCGG GAAATTGTGA

51

GGgACATGAA GgAGAAgCTG TCCTACATTG CCCTGGACTA TGACCAgGAA

101 ATgGagActT CTAAgACCAg CTCATCTgTT GAGAAgAgct ACGAgctTCC

151 TGATGgGCAA GTTATCACCA TTGGTgCTGA GCGTTTCCGT TGCCCTGAgG

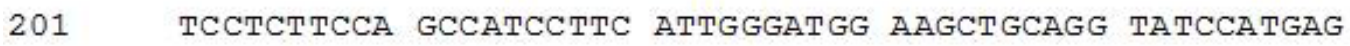

251 ACCACCTACA ACTCCATCAT GAAGTGCGAC GTgAAtATtA GgAaggatct

301 ATACGgCAAC ATTGTTCTTA GTGGTgGTAC CACtATGTTC ACTgGAATtg

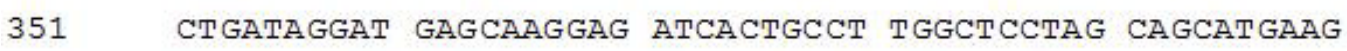

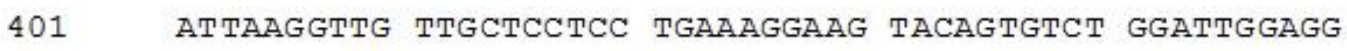

$451 \quad$ TGC

Figure 6. Actin gene sequence.

\section{Expression levels of B231P5CS}

RT-qPCR was performed to determine the expression patterns of B231P5CS in response to salt stress. The transcript level of B231P5CS remained low and constant in both shoots and roots in the absence of stress treatment (Figure 7A and B), especially in roots. The transcript level of $B 231 P 5 C S$ in roots was much lower than that in shoots. The transcript levels of $B 231 P 5 C S$ genes began to increase in leaves after $2 \mathrm{~h}$ of stress treatment. After treatment for $4 \mathrm{~h}$, expression was lower than at $2 \mathrm{~h}$, but higher than that of the control group. Thereafter, the transcription level of B231P5CS continued to increase, peaking at $12 \mathrm{~h}$. In the leaves of salt-stressed plants, B231P5CS transcripts increased nearly 15-fold relative to the controls. Such a large induction of $B 231 P 5 C S$ suggests that it plays a primary role in proline accumulation during prolonged stress. In contrast, the transcript level of B231P5CS in roots decreased moderately in plants treated with salt. The leaves of RCG have a tendency to maintain or increase P5CS activity as a consequence of salt treatment, but high salt concentration (200 mM $\mathrm{NaCl}$ ) also induced a decline in the transcription of B231P5CS in roots. 

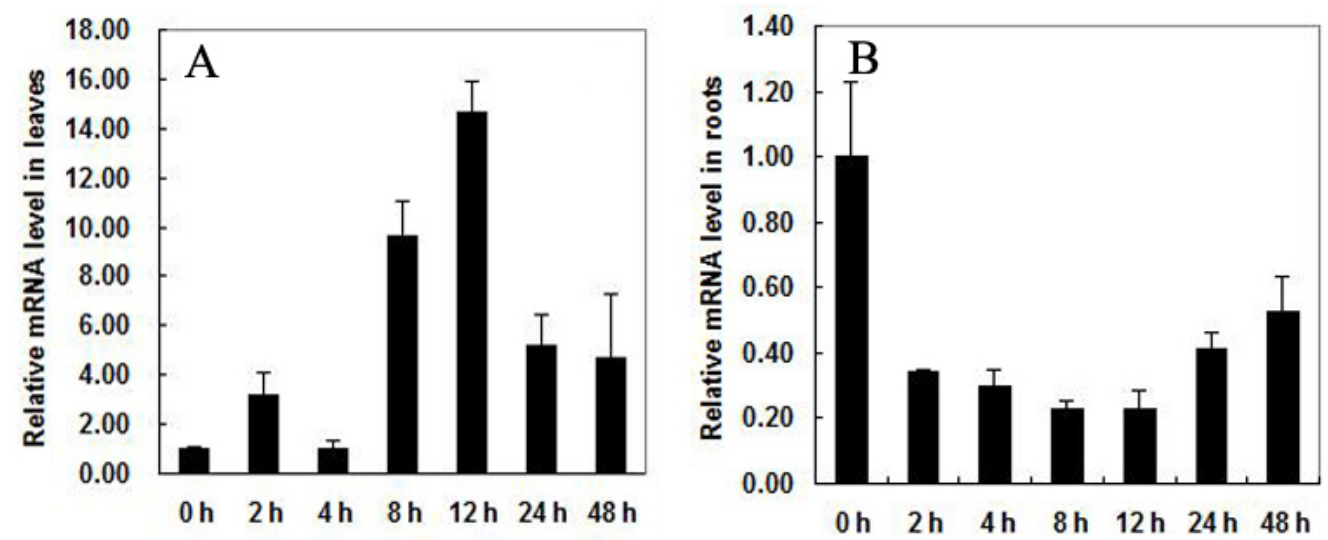

Figure 7. Relative mRNA levels in roots and leaves of reed canary grass after $0,2,4,8,12,24$, and $48 \mathrm{~h}$ exposure to $200 \mathrm{mM} \mathrm{NaCl}$ (A. leaves; B. roots).

\section{Proline content}

To test whether proline accumulation is correlated with the expression level of B231P5CS genes, proline content was measured in the same samples used for RT-PCR assays. Figure 8 shows that under non-stress conditions, proline levels were low in both the shoots and roots of RCG. The expression level of proline in leaves was almost 20-fold higher than that in the roots of plants not under salt stress. We can deduce that proline mainly accumulates in leaves under normal growth conditions. After $8 \mathrm{~h}$ of salt stress, proline content increased sharply in leaves and peak proline content was detected after $12 \mathrm{~h}$ of salt stress. Peak proline concentration was approximately 2-fold higher than that of the controls (Figure 8A). In contrast, proline content decreased in roots after salt treatment (Figure 8B). Relative to control roots, there was a 3.6-fold decrease in proline concentration after $8 \mathrm{~h}$ of salt stress treatment and a 3.2 -fold decrease after $12 \mathrm{~h}$ of salt stress treatment.
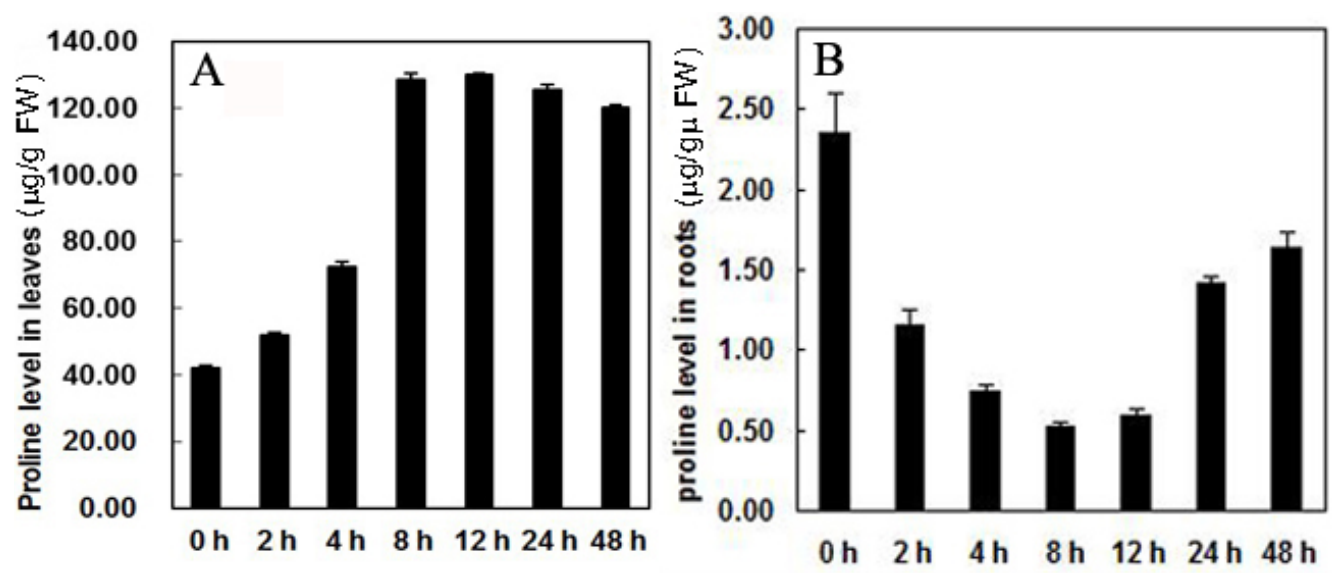

Figure 8. Proline content in leaves $(\mathbf{A})$ and roots $(\mathbf{B})$ of reed canary grass seedlings. 


\section{DISCUSSION}

Accumulation of proline is an important indicator of salt stress tolerance in higher plants (Hur et al., 2004). Salt stress increases the accumulation of proline in numerous plant species and it may enhance osmotic stress tolerance (Sawahel and Hassan, 2002; Silva-Ortega et al., 2008). Proline is one of the most accumulated osmolytes found in plants grown in high salinity or water deficit conditions (Delauney et al., 1993; Jimenez-Bremont et al., 2006; Tripathi et al., 2007) and is believed to play an adaptive role in plant stress tolerance (Yancey et al., 1982; Delauney et al., 1993). P5CS is a bifunctional enzyme that catalyzes the first two steps of proline biosynthesis in plants. It is a rate-limiting enzyme in this pathway and exhibits both $\gamma$-glutamyl kinase and glutamic- $\gamma$-semialdehyde dehydrogenase activity (Kavi Kishor et al., 1995). To date, many P5CS orthologs have been cloned from various plant species, but there have been no reports of cloned P5CS from RCG. In the present study, we isolated and characterized a P5CS gene in RCG. B231P5CS may have roles similar to those of previously characterized $P 5 C S$ genes of other species.

In higher plants, proline accumulation appears to be due to de novo synthesis (Peng et al., 1996). Catabolism rate minimization, decreased proline utilization in protein synthesis, and increased proteolysis may also contribute to net proline accumulation (Dungey and Davies, 1982; Forlani et al., 1997). P5CS, which catalyzes the first two steps in proline biosynthesis, is critical for the control of proline biosynthesis and, consequently, an increase in osmotolerance. This essential regulatory function probably occurs at two levels of control: proline feedback inhibition on the P5CS protein and regulation of the P5CS transcript level in response to osmotic stress conditions (Jimenez-Bremont et al., 2006).

In plants, the process of proline biosynthesis is orderly and complex, and many factors are involved (Kishor et al., 2005). In the present study, two important factors, free proline content and the mRNA level of B231P5CS, were determined in RCG. We found that the P5CS gene and proline accumulation were not always induced by high salt stress. For example, $P 5 C S$ expression and proline content increased in leaves while expression levels decreased in roots. Although $3231 P 5 C S$ expression and proline accumulation differ among tissues, the proline level in stressed plants is associated with B231P5CS expression. P5CS expression and proline content are reported to vary among different Arabidopsis organs (Verbruggen et al., 1993; Chiang and Dandekar, 1995). Previous studies indicate a positive relationship between P5CS, proline accumulation and plant stress tolerance. Proline accumulation during osmotic stress is mainly due to increased synthesis and reduced degradation (Verbruggen and Hermans, 2008). The proline degradation process is the reverse of proline biosynthesis and is catalyzed by proline dehydrogenase $(\mathrm{PDH})$ and $\mathrm{P} 5 \mathrm{C}$ dehydrogenase $(\mathrm{P} 5 \mathrm{CDH})$. Reciprocal regulation of P5CS and PDH genes plays a key role in the control of proline levels during and after osmotic stress (Szekely et al., 2008) Therefore, proline accumulation may result from damage to proteins that regulate the expression of $P D H$ or $P 5 C D H$ genes as a result of abiotic stresses. In Arabidopsis, both transcript and protein levels of AtPDH decreased during stress and increased during recovery from stress (Peng et al., 1996), indicating that proline level was partly affected by $A t P D H$. It has also been reported that proline content is related to transport processes in plants under stress (Lehmann et al., 2010).

Transgenic approaches to improving plant stress tolerance via the overproduction of proline have been successful. For example, overexpression of P5CS in tobacco (Kavi Kishor et al., 1995) and Arabidopsis (Nanjo et al., 2003) significantly enhances stress tolerance. In 
the present study, the RCG B231P5CS gene was isolated and characterized, and its expression under stress conditions was examined. B231P5CS may be a stress-inducible gene that regulates the accumulation of proline in the leaves of plants subjected to salt stress. Further experiments, including overexpression of this gene using transgenic approaches may advance our understanding of the role of B231P5CS in stress tolerance.

\section{ACKNOWLEDGMENTS}

Research supported by the Ministry of Science and Technology, China (grant Nos. \#2012AA101801, \#2011AA100209, \#2012CB215300, and \#2009BADA7B04); the Scientific Research Fund of China Agricultural University (grant No. \#2014FG062), and the National Natural Science Fund Committee, and Industry \& Technology System (forage) (grant No. \#31272493). We appreciate the generous support given by Dr. MeiYan Zhang. We also thank Yongxiang Li for his assistance in sample collection and processing, as well as offering interesting discussions.

\section{REFERENCES}

Anderson GQA and Fergusson MJ (2006). Energy from biomass in the UK: sources, processes and biodiversity implications. Ibis 148: 180-183.

Anoop N and Gupta AK (2003). Transgenic indica rice cv IR-50 overexpressing Vigna aconitifolia delta 1-pyrroline-5carboxylate synthetase cDNA shows tolerance to high salt. J. Plant Biochem. Biot. 12: 109-116.

Borjesson P (1999). Environmental effects of energy crop cultivation in Sweden-II: Economic Valuation. Biomass Bioenerg. 16: 155-170.

Chiang HH and Dandekar AM (1995). Regulation of proline accumulation in Arabidopsis thaliana (L.) Heynh during development and in response to desiccation. Plant Cell Environ. 18: 1280-1290.

Delauney A J and Verma DPS (1993). Proline biosynthesis and osmoregulation in plants. Plant J. 4: 215-223.

Delauney AJ, Hu CA, Kishor PB and Verma DPS (1993). Cloning of ornithine daminotransferase cDNA from Vigna aconitifolia by trans-complementation in Escherichia coli and regulation of proline biosynthesis. J. Biol. Chem. 268: 18673-18678.

Dungey NO and Davies DD (1982). Protein turn over in isolated barley leaf segments and the effects of stress. J. Exp. Bot. 33: 12-20.

Finell M and Nilsson C (2005). Variations in ash content, pulp yield, and fiber properties of reed canary-grass. Ind. Crop Prod. 22: 157-167.

Forlani G, Scainelli D and Nielsen E (1997). [delta]1-Pyrroline-5-Carboxylate dehydrogenase from cultured cells of potato (purification and properties). Plant Physiol. 113: 1413-1418.

Hmida-Sayari A, Gargouri-Bouzid R, Bidani A, Jaoua L, et al. (2005). Overexpression of $\Delta$ 1-pyrroline -5-carboxylate synthetase increases proline production and confers salt tolerance in transgenic potato plants. Plant Sci. 169: 746752.

Hu CAA, Delauney AJ and Verma DPS (1992). A bifunctional enzyme $\Delta$ 1-pyrroline -5- carboxylate synthetase) catalyzes the first two steps in proline biosynthesis in plants. Proc. Natl. Acad. Sci. U. S. A. 89: 9354-9358.

Hur J, Jung KH, Lee CH and An G (2004). Stress-inducible OsP5CS2 gene is essential for salt and cold tolerance in rice. Plant Sci. 167: 417-426.

Jimenez-Bremont JF, Becerra-Flora A, Hernandez-Lucero E, Rodriguez-Kessler M, et al. (2006). Proline accumulation in two bean cultivars under salt stress and the effect of polyamines and ornithine. Biol. Plantarum 50: 763-766.

Kavi Kishor PB, Hong Z, Miao GH, Chein-An A, et al. (1995). Overexpression of [delta]-pyrroline-5- carboxylatesynthetase increases proline production and confers osmotolerance in transgenic plants. Plant Physiol. 108: 13871394.

Kishor K, Sangam S, Amrutha RN, Laxmi PS, et al. (2005). Regulation of proline biosynthesis, degradation, uptake and transport in higher plants: Its implications in plant growth and abiotic stress tolerance. Curr. Sci. India 88: 424-438.

Landstrom S, Lomakka L and Anderson S (1996). Harvest in spring improves yield and quality of reed canary grass as a bioenergy crop. Biomass. Bioenerg. 11: 333-341. 
Latef AAA (2010). Changes of antioxidative enzymes in salinity tolerance among different wheat cultivars. Cereal Res. Commun. 38: 4355.

Lehmann S, Funck D, Szabados L and Rentsch D (2010). Proline metabolism and transport in plant development. Amino Acids 39: 949-962.

Lewandowski I, Scurlock J, Lindvall E and Christou M (2003). The development and current status of perennial rhizomatous grasses as energy crops in the US and Europe. Biomass. Bioenerg. 25: 335-361.

Livak KJ and Schmittgen TD (2001). Analysis of relative gene expression data using real-time quantitative PCR and the 2(-Delta Delta C(T)) method. Methods 25: 402-408.

Magne C and Larher F (1992). High sugar content of extracts interferes with colorimetric determination of amino acids and free proline. Anal. Biochem. 200: 115-118.

Nanjo T, Fujita M, Seki M, Kato T, et al. (2003). Toxicity of free proline revealed in an arabidopsis T-DNA-tagged mutant deficient in proline dehydrogenase. Plant Cell Physiol. 44: 541-548.

Peng Z, Lu Q and Verma DP (1996). Reciprocal regulation of delta 1-pyrroline-5-carboxylate synthetase and proline dehydrogenase genes controls proline levels during and after osmotic stress in plants. Mol. Gen. Genet. 253: 334-341.

Sahramaa M (2004). Evaluating Germplasm of Reed Canary Grass, Phalaris arundinacea. University of Helsinki, Helsinki.

Sahramaa M and Jauhiainen L (2003). Characterization of development and stem elongation of reed canary grass under northern conditions. Ind. Crop Prod. 18: 155-169.

Savoure A, Jaoua S, Hua XJ, Ardiles W, et al. (1995). Isolation, characterization, and chromosomal location of a gene encoding the delta 1-pyrroline-5-carboxylate synthetase in Arabidopsis thaliana. FEBS Lett. 372: 13-19.

Sawahel WA and Hassan AH (2002). Generation of transgenic wheat plants producing high levels of the osmoprotectant proline. Biotechnol. Lett. 24: 721-725.

Sheng M, Tang M, Chen H, Yang B, et al. (2008). Influence of arbuscular mycorrhizae on photosynthesis and water status of maize plants under salt stress. Mycorrhiza 18: 287-296.

Silva-Ortega CO, Ochoa-Alfaro AE, Reyes-Aguero JA, Aguado-Santacruz GA, et al. (2008). Salt stress increases the expression of $\mathrm{p} 5 \mathrm{cs}$ gene and induces proline accumulation in cactus pear. Plant Physiol. Biochem. 46: 82-92.

Sripinyowanich S, Klomsakul P, Boonburapong B, Bangyeekhun T, et al. (2013). Exogenous ABA induces salt tolerance in indica rice (Oryza sativa L.): The role of OsP5CS1 and OsP5CR gene expression during salt stress. Environ. Exp. Bot. 86: 94-105.

Szabados L and Savoure A (2010). Proline: a multifunctional amino acid. Trends Plant Sci. 15: 89-97.

Szekely G, Abraham E, Cseplo A, Rigo G, et al. (2008). Duplicated P5CS genes of Arabidopsis play distinct roles in stress regulation and developmental control of proline biosynthesis. Plant J. 53: 11-28.

Tripathi SB, Gurumurthi K, Panigrahi AK and Shaw BP (2007). Salinity induced changes in proline and betaine contents and synthesis in two aquatic macrophytes differing in salt tolerance. Biol. Plantarum 51: 110-115.

Vepsalainen V (2010). Energy crop cultivations of reed canary grass-An inferior breeding habitat for the skylark, a characteristic farmland bird species. Biomass. Bioenerg. 34: 993-998.

Verbruggen N and Hermans C (2008). Proline accumulation in plants: a review. Amino Acids 35: 753-759.

Verbruggen N, Villarroel R and Van Montagu M (1993). Osmoregulation of a pyrroline-5-carboxylate reductase gene in Arabidopsis thaliana. Plant Physiol. 103: 771-781.

Yancey PH, Clark ME, Hand SC, Bowlus RD, et al. (1982). Living with water stress: evolution of osmolyte systems. Science 217: 1214-1222.

Zhang CS, Lu Q and Verma DP (1995). Removal of feedback inhibition of delta 1-pyrroline-5-carboxylate synthetase, a bifunctional enzyme catalyzing the first two steps of proline biosynthesis in plants. J. Biol. Chem. 270: 20491-20496. 